\title{
Essai de lutte contre Glossina fusca, par pulvérisation de dieldrin, en République Centrafricaine
}

\author{
par P. FINELLE, J. DESROTOUR, P. YVORÉ, P. RENNER*
}

Le Dieldrin est utilisé dans la lutte contre les glossines depuis 1955 et plusieurs campagnes ont été réalisées, surtout dans les pays de langue anglaise, tant contre Glossina palpalis ou G. fuscipes (Kenya : BURNETT et Coll.,1957; GLOVER ef Coll., 1958. Nigeria : DAVIES, 1960 ; MAHOOD, 1960 ; Uganda: HOCKING, 1961) que contre G. pallidipes (Kenya : THOMPSON et Coll., 1960). Au Nord Cameroun, une expérience a été réalisée en 1961 contre G. tachinoïdes (MOUCHET et Coll.).

Les résultats obtenus par ces divers essais ont incité le service de l'Elevage de la République Centrafricaine en collaboration avec l'Institut d'Elevage et de Médecine vétérinaire des Pays tropicaux, à tenter une opération analogue mais dirigée contre une espèce différente : $G$. fusca.

Le financement de cette campagne de lutte a été entièrement assuré par le fond d'aide et de coopération.

\section{1. - LES PROBLÈMES}

\section{Aspect géographique et économique}

La région choisie pour ce premier essai se trouve au Nord-Ouest de la République Centrafricaine à proximité de la frontière du Cameroun, dans le district de Babova: (Carte l) le bi: recherché était l'assainissement de ic partie supérieure du cours de la rivière Nié.

\footnotetext{
* Nous tenons d̀ remercier MM. BAiLLY, RAY vaUD ef LEAUTE qui, successivement, ont collaboré à cette campagne.

Rev. Elev. Méd. vét. Pays trop. 1962, 15, no 3.

Reçu pour publication : juillet 1962.
}

Cette région, située entre 950 eł: 1.100 mètres d'altitude est couverte de savanes arborées à dominance de Burkea africana et Lophira alata, coupées de galeries forestières; la pluviométrie atteint en moyenne $1.400 \mathrm{~mm}$ ef le réseau hydrographique y est très dense. Elle borde au sud la zone occupée par le bétail en saison des pluies et, en saison sèche, elle constitue une zone de passage, empruntée par les troupeaux allant vers le Sud à la recherche de pâturages plus humides. Pendant la saison des pluies, elle est totalement désertée alors qu'il y a seulement une dizaine d'années de nombreux troupeaux y séjournaient : la dégradation des pâturages, très rapide dans cette région à forte pluviométrie et à relief très vallonné, a été la cause essentielle de cet abandon, mais les pertes causées par la trypanosomiase, pratiquement inconnue autrefois, y ont certainement fortement contribué.

\section{$2^{\circ}$ Aspect entomologique}

La répartition des glossines est donnée dans la carte II. On y voit que $G$. fuscipes fuscipes Newst. n'est présente que le long des rivières importantes: la Nana et la Mambéré, et qu'elle disparaît lorsque les rivières s'amenuisent. Par contre G. fusca congolensis Newst. et Evans. est beaucoup plus largement répartie et se rencontre dans les galeries forestières, souvent importanies, qui bordent les ruisseaux et rivières de faible débit. G. fuscipleuris Austen coexiste souvent avec $G$. fusca mais est toujours beaucoup plus rare.

Dans le cas particulier de la Mambéré, $G$. fuscipes ne semble remonter qu'à une quinzaine I de kilomètres au nord du village de Baboua 
(altitude 900 mètres environ) et aucune glossine n'a pu être trouvée le long du cours supérieur de cette rivière. En ce qui concerne la Nie, $G$. fusca y a été trouvée, légèrement au nord du village de Gadze, ce qui correspond environ à une altitude de 1.000 mètres.

Plusieurs faits incitent à penser que la présence des glossines le long du cours supérieur de la Nie est récente ou tout au moins que leur densité apparente a nettement augmenté ces dernières annèes :

- Apparition récente des trypanosomiases animales.

- En 1955, aucune glossine n'avait pu être trouvée bien que les prospections aient eu lieu au mois de mai, soit au début de saison des pluies, période où les glossines sont généralement abondantes.

- La première G. fusca a été capturćc en 1958 et depuis nous en avons régulièrement trouvé, bien que toujours en faible nombre.

Le but de l'opération de lutte décrite dans cette note, a donc été non seulement de récupérer une zone contaminée par $G$. fusca mais surtout de créer une barrière s'opposant à la remontée de cette glossinc et ćgalement, on prévision d'opérations futures de plus grande envergure, d'entraîner le personnel à l'emploi des appareils et de préciser le prix de revient moyen, en fonction des conditions locales.

\section{II. - LA RÉALISATION}

\section{Io Bases biologiques de la lutte contre G. fusca}

Le principe de la lutte contre $G$. fusca est fondé sur deux faits essentiels de la biologie de cette glossine :

- La présence de G. fusca est liée à la forêt ; si parfois on peut la rencontrer en savane, c'est toujours à faible distance d'une zone forestière et il semble bien que cette glossine ne puisse parcourir que, de faibles distances en terrain dégagé (Nous ne l'avons jamais observée à plus d'un kilomètre d'une forêt).

- Les lieux de repos de G. fusca sont constitués par des lianes, des arbustes, des troncs d'arbres de faible dimension, généralement à une hauteur inférieure à $2 \mathrm{~m}$.

Une expérience réalisée à la station de Bewiti, portant sur 500 glossines, a montré que:
- $95 \%$ des mouches se posent sur des supports dont le périmètre est compris entre 0 ef $40 \mathrm{~cm}$ ef ce périmètre n'a jamais été supérieur à $90 \mathrm{~cm}$ (graphique I).

- $96 \%$ se posent à des hauteurs comprises entre $60 \mathrm{~cm}$ et $2 \mathrm{~m}$.

Aucune glossine n'a été observée posée à une hauteur supérieure à 2,20 m (graphique II).

En fonction de ces données, pour assainir une région il doit suffir :

a) d'isoler le cours supérieur d'une rivière en constituant en aval une barrière infranchissable par les glossines, c'est-à-dire en abattant la galerie forestière sur une distance suffisante : pour ce premier essai et faute de données ṕlus précises, nous avons choisi arbitrairement' une distance en ligne droite d'environ 3 kilomètres.

b) de traiter, en saison sèche, les lieux de repos des glossines avec un insecticide dont la rémanence soit supérieure à la durée d'éclosion des pupes (au maximum 1 mois) : d'après les travaux des auteurs anglais le Dieldrin utilisé en pulvérisation à $2 \%$ remplit largement cés conditions, s'il est employé en pleine saison sèche de manière à ce que l'insecticide persiste sur les végétaux et ne soit pas lavé par les pluies (d'après DAVIES, 1960 , le Dieldrin, en pulvérisation à $4 \%$, garderait son activité pendant plus d'un an).

\section{Exécution des travaux}

\section{a) Prospections préliminaires}

Les prospections préliminaires avaient pour but de déterminer avec précision l'aire d'extension de G. fusca, de situer le meilleur emplacement pour la réalisation de la coupure et les limites de la zone à pulvériser (carte III). Ces études ont été faites sur le terrain et sur photos aériennes.

\section{b) Abattage des galeries forestières}

L'emplacement de la coupure a été choisi de part et d'autre de la route Baboua-Besson, tant pour des raisons de facilité d'accès que parce que cette zone était déjà partiellement débroussée, et mise en culture par les habitants du village de Gadze. Par contre le choix de cet emplacement obligeait à effectuer l'abattage de la galerie forestière d'un petit affluent de la Nie, en plus de celui de la galerie principale. 
Par mesure de précaution une autre coupure a été réalisée sur la Mambéré de manière à empêcher une éventuelle remontée de glossines le long de ceffe rivière.

En tout, environ 8 kilomètres de galeries forestières ont été abattues. Leurs largeurs étaient très variables: en certain point où les rives étaient escarpées elles ne dépassaient pas une vingtaine de mètres, alors que dans certaines zones marécageuses elles en atteignaient 200. On peut approximativement évaluer la superficie qui a été déboisée à une cinquantaine d'hectarès.

Le travail débuta en août 1960 et fut terminé en mars 1961 mais il yeut plusieurs périodes d'arrêt et la durće effective des travaux n'excéda pas 4 mois.

Aucun moyen mécanique ne fut utilisé et seuls furent employés les outils traditionnels: haches, coupe-coupes, scies. Les arbres abattus ainsi que les broussailles furent brôlés sur place de manière à dégager le terrain en vue de sa mise en culture ultérieure.

\section{c) Traitement insecticide}

\section{1. - L'insecticide}

Nous avons utilisé du «Dieldrex CE 20», liquide concentré émusifiable, contenant 20 p. 100 de dieldrin, qui pour l'emploi était dilué au $10 \mathrm{e}$ de manière à obtenir une concentration de 2 p. 100 en produit actif. Pour la facilité des manutentions nous avons choisi la présentation en bidons métalliques de 20 litres : en tout 600 litres de concentré furent utilisés.

\section{2. - Les pulvérisateurs}

Pour ce premier essai réalisé en République Centrafricaine plusieurs types d'appareils ont été essayés de manière à déterminer le modèle convenant le mieux à ce travail particulier.

Nous avons choisi des pulvérisateurs à dos, à pression préalable. Quatre modèles ont été retenus (le choix n'a pas été fait d'une manière délibérée, mais en fonction des appareils disponibles sur le marché local). litres.

— «Vermorel» type «colibri», capacité utile 14

— «Galeazzi » capacité utile 12 litres.

- «Muratori » grand modèle, capacité utıle 12,5 litres.

- «Muratori » petit modèle, capacité utıle 10 litres.
Tous ces divers modèles d'appareil ont donné satisfaction par leur robustesse ef leur facilité d'emploi. Mais, éłant donné les conditions très dures de travail (pulvérisation en galeries forestières souvent très touffues, plus ou moins marécageuses... etc), ces appareils légers semblent préférables, la faible contenance n'étant qu'un inconvénient minime puisque le travail a toujours lieu à proximité d'un cours d'eau.

12 pulvérisateurs ont été utilisés (4 de chaque marque) et tous étaient encore en état de marche après la fin des opérations bien qu'aucun personnel spécialisé n'ait été utilisé pour les pulvérisations.

\section{3. - Pulvérisation}

La zone où les pulvérisations ont été faites a été déterminée en tenant compte de la répartition connue de $G$. fusco et en débordant très largement, de manière à ménager une large marge de sécurité (carte III).

12 équipes de 3 hommes furent utilisées, chacune possédant un pulvérisateur. L'un des hommes actionnait le pulvérisateur, le deuxième portait la réserve d'insecticide concentré, le troisième préparait le chemin au moyen d'un coupe-coupe.

En galcrie forestière moyenne (une cinquantaine de mètres de large) 4 équipes travaillaient parallèlement, 2 longeant la rivière pulvérisaient l'un la rive gauche, l'autre la rive droite, les deux autres équipes avançaient dans la galerie elle-même. Dans ces conditions 20 litres de concentré ont été en moyenne utilisés par kilomètre de galerie forestière.

En fait, suivant la largeur de la forêt le nombre des équipes utilisées varia entre 2 et 8 . Au total une cinquantaine de kilomètres de forêt fut ainsi traitée.

$$
\text { 4. - Coût }
$$

Le coût total de cette campagne a été de $1,780.000 \mathrm{fr}$ C. F. A. qui se décomposent ainsi :
Photos aériennes, cartes........... $\quad 45.000$
Prospection entomologique ........ 40.000
Main-d'œurre employée au déboise-
ment ..................... 600.000
Main-d'cuvre employée d̀ la pulvéri-

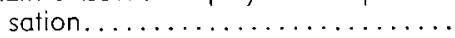

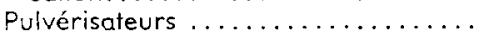

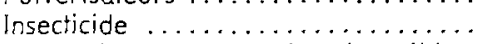
Divers (essence, entretien des véhicu-
les et du matériel) ...............
Personnel d'encadrement ............ $\quad 300.000$
$\overline{1.780 .000}$ fr. CFA 
D'après ces données on peut évaluer, par km linéaire de galerie forestière, le prix de revient des déboisements à environ $110.000 \mathrm{fr}$. et celui des pulvérisations à 15.000 fr. On doit noter cependant que las pulvćrisatcurs inscrits en dépense restent utilisables pour des opérations ultérieures.

\section{III. - LES RÉSULTATS}

Des prospections ont été faites dans la zone traitée ainsi qu'en aval de la coupure de manière à avoir un élément de comparaison. Elles seront poursuivies encore pendant plusieurs mois.

8 mois après la fin des pulvérisations et après une saison des pluies complète, aucune glossine n'a été trouvée dans la zone traitée. Par contre en aval de la coupure, des glossines ont régulièrement été capturées. L'opération semble donc réussie mais il est évidemment encore beaucoup trop tôt pour conclure définitivement.

Après ce premier succès l'important est évidemment de maintenir l'efficacité de la barrière constituée par les parties déboisées : l'idéal serait que ces zones soient intégralement mises en cultures ce qui résoudrait le problème. A défaut, il sera, dans l'avenir, indispensable de détruire périodiquement la végétation arbustive au fur et à mesure qu'elle réapparaîtra.

En fonction des résultats et des enseignements fournis par ce premier essai, une nouvelle campagne de beaucoup plus grande envergure a été entreprise dans la sous-préfecture de 'Carnot. Elle porte sur une zone infestée par $G$. fuscipes et est actuellement en cours de réalisation.

Instifut d'Elevage ef de Médecine vétérinaire des Pays tropicaux

Centre de recherche sur les trypanosomiases animales de Bouar

Service de l'Elevage de la République Contrafricainc.

\section{RÉSUMÉ}

La technique utilisée consiste à isoler la partie supérieure d'une galerie forestière en effectuant un déboisement de 3 kilomètres ef à traiter la zone ainsi isolée en pulvérisant du Dieldrin à 2 p. 100 sur les lieux de repos de $G$. fusca.

Les premiers résultats semblent indiquer la réussite de cette opération. Pour l'avenir,' le principal problème est le maintien de la zone déboisée.

\section{SUMMARY}

\section{Trials to Eradicate Glossina fusca in the Central African Republic by spraying with Dieldrin}

The technique consists in isolating the upper part of a forest gallery by clearing an area of $3 \mathrm{Km}$ of trees and, having thus isolated it, spraying the resting places therein of $G$. fusca with dieldrin at $2 \%$.

The first results obtained seem to indicate that this operation is a success. The main problem in the future will be the maintenance of clearing in this area.

\section{RESUMEN}

\section{Experimento de lucha contra Glossina fusca mediante pulverización de Dieldrín en la República Centroafricana.}

La téenica utilizada consiste en aislar la parte superior de una galeria florestal efectuando una tala de 3 kilómetros y tratar la zona asi aislada pulverizando Dieldrin al $2 \%$ en los lugares de reposo de la G. fusca.

Los primeros resultados parecen indicar el éxito de esta operación. En lo futuro, el principal problema consiste en el mantenimiento de la zona de desmonte. 


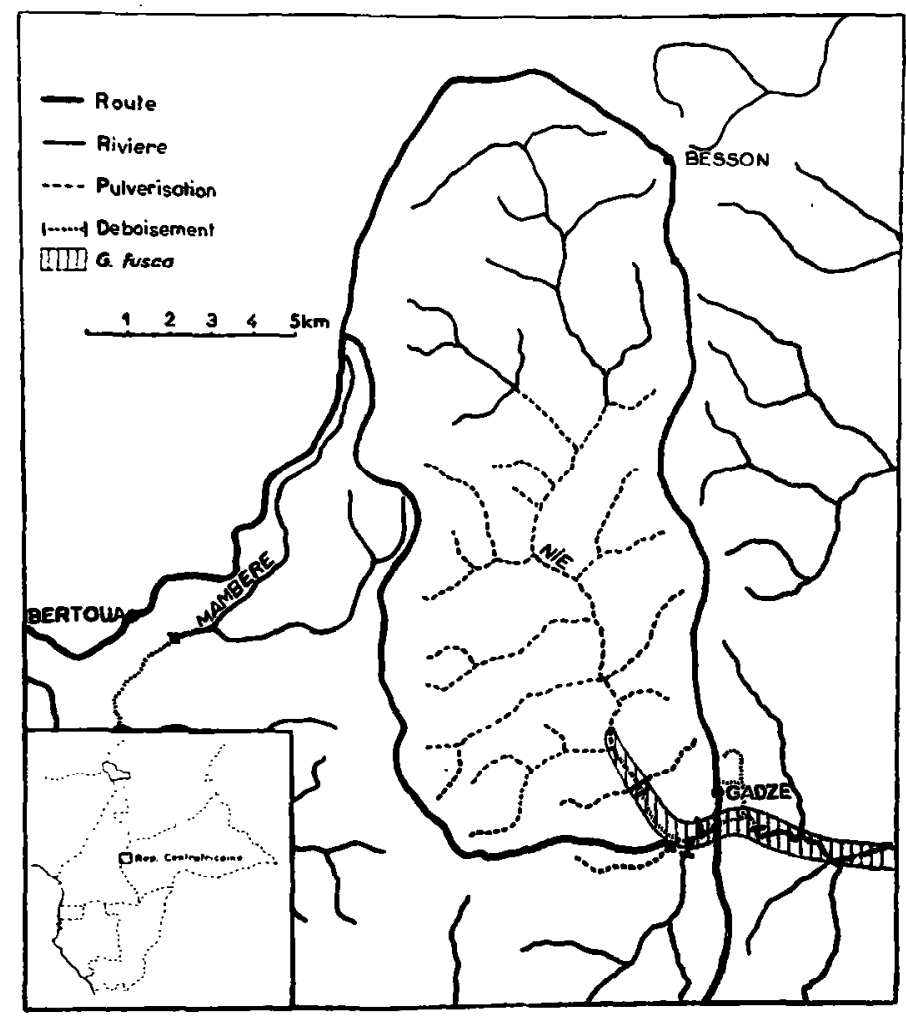

Carte I. - Situation géographique.

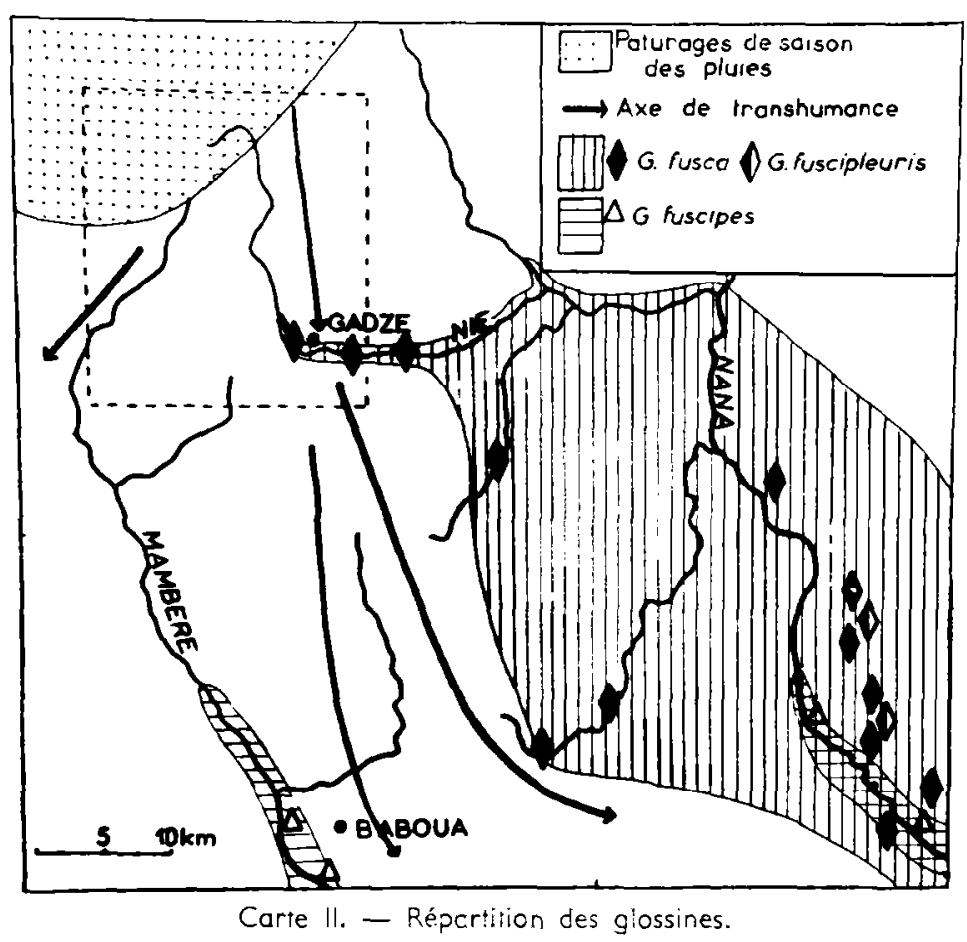




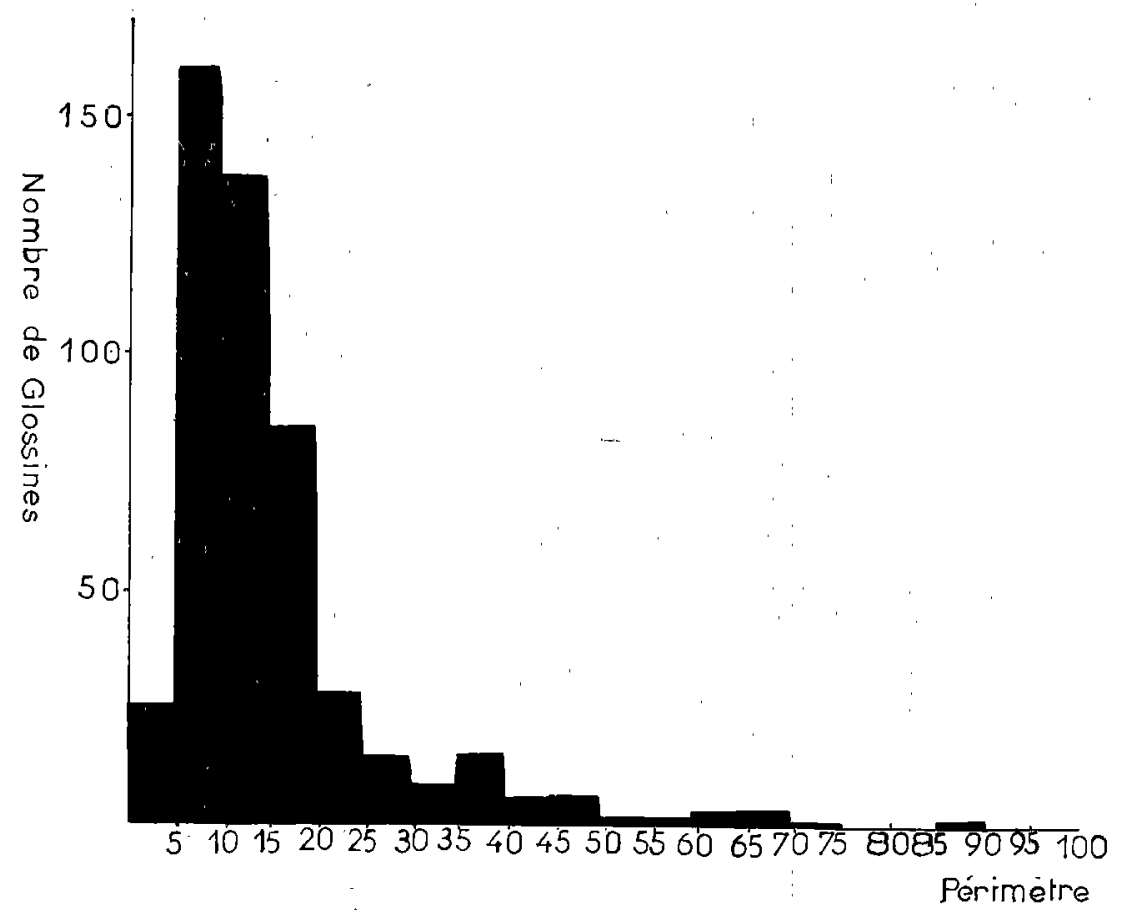

Tableau 1. - Lieu de repos de G. Fusca. Périmètre du tronc.

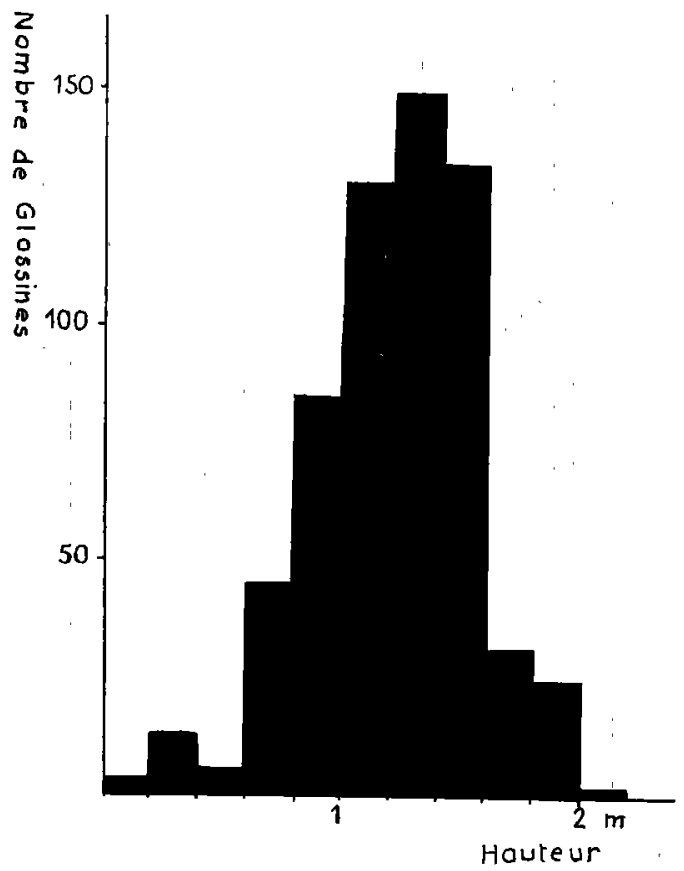

Tableau 1I. - Lieu de repos de G. Fusca. Hauteur. 


\section{BIBLIOGRAPHIE}

BURNETT (G.F.), ROBINSON (J.) et LE ROUX (J. G.). - Comparative trials of D.D.T. and Dieldrin for the control of riverside tsetse Glossina polpalis fuscipes Newst. E. Afric. Agric. J. 1957, 22 : 142-6.

DAVIES (J. B.). - Dieldrin in the control of Glossina palpalis (R. D.) in the Southern Guinea savannah of N. Nigeria. Com. Scient. Inter. Rech. Trypano. Jos 1960, 277-84.

FARRELL (J. A. K.). - The control of a tsetse fly (Glossina) advance by use of residual insecticide. Com. Scient. Intern. Rech. Trypano. Jos 1960, 265-8.

GLOVER (P. E.), LE ROUX (J. G.) et PARKER (D.F.). - The extermination of Glossina palpalis on the Kuja-Migori river systems with the use of insecticides. Com. Scient. Intern. Rech. Trypano. Bruxellcs 1958, 331-42.

GLOVER (P. E.) et TRUMP (E. C.). - La lutte contre la mouche tsétsé au Kenya. Aspects économiques. Span 1960, 3 (3) : 109-13.
HOCKING (K.S.). - Discriminative application of insecticide against Glossina morsitans West. Bull. Entom. Res. 1961, 52 (1) : 17-22. MAC DONALD (W. A.). - Insecticidal spraying against Glossina palpalis in Nigeria based on a study of its nocturnal resting sites with ultra-violet light. Com. Scient. Intern. Rech. Trypano. Jos 1960, 243-5.

MAHOOD (A.). - An experiment on the control of Glossina palpalis R. D. in Southern Zaria province, N. Nigeria, by chemical means. Com. Scient. Intern. Rech. Trypano. Jos 1960, 269-76.

MOUCIIET (J.), DELAS (A.) ct YVORE (P.). La campagne expérimentale de lutte contre Glossina tachinoïdes West, à Logone-Birni (Rép. du Cameroun et Rép. du Tchad). Bull. Soc. Path. Exot. 1961, 54 (4) : 875-92.

PAGE (W. A.) - Some obscrvations on the fusca group of tsetse flies in the South of Nigeria. Bull. Entom. Res. 1959, 50 (3) : 633-46. 